\title{
Distributed Measurements for Estimating and Updating Cellular System Performance
}

\author{
Liang Xiao, Student Member, IEEE, Larry J. Greenstein, Life Fellow, IEEE, \\ Narayan B. Mandayam, Senior Member, IEEE, and Shalini Periyalwar, Senior Member, IEEE
}

\begin{abstract}
We investigate the use of distributed measurements for estimating and updating the performance of a cellular system. Specifically, we discuss the number and placement of sensors in a given cell for estimating its signal coverage. Here, an "outage" is said to occur at a location if a mobile receiver there has inadequate signal-to-noise ratio ( $S N R$-based outage) or, using another criterion, inadequate signal-to-interference ratio (SIRbased outage); and the "outage probability" is the fraction of the cell area over which outage occurs. A design goal is to improve measurement efficiency (i.e., minimizing the required number of measurement sensors) while accurately estimating the outage probability and mapping the coverage holes. The investigation uses a generic path loss model incorporating distance effects and spatially correlated shadow fading. Our emphasis is on the performance prediction accuracy of the sensor network, rather than on cellular system analysis per se. Through analysis and simulation, we assess several approaches to estimating the outage probability. Applying the principle of importance sampling to the sensor placement, we show that a cell outage probability of $P_{o}$ can be accurately estimated using $\sim 10 / P_{o}$ power-measuring sensors distributed in a random uniform way over the area with basesensor distances from $50 \%$ to $100 \%$ of the cell radius. This result applies to both $S N R$-based and SIR-based outage estimation for both indoor and outdoor environments.
\end{abstract}

Index Terms-Distributed measurements, cellular systems, importance sampling, sensor networks, path loss.

\section{INTRODUCTION}

W E investigate the use of distributed measurements in a cell via sensors at known locations. The aim is to assist radio resource management in maximizing the quality of service in cellular systems. Our focus is on new or envisioned cellular system designs in which antenna beams, power per beam and channel sets can be assigned adaptively to accommodate slowly changing conditions of the propagation and the user population. The sensor-measured data can reduce the measurement demands on the active mobiles; or, it can be augmented by such measurements, to permit more dynamic adapting as individual mobiles change locations, start and end service, and so on.

Paper approved by K. K. Leung, the Editor for Wireless Network Access and Performance of the IEEE Communications Society. Manuscript received May 25, 2006; revised March 15, 2007. This work was supported in part by a grant from Nortel. This paper was presented in part at the IEEE International Conference on Communications, June 2006, Istanbul, Turkey.

L. Xiao, L. Greenstein, and N. Mandayam are with WINLAB, the Department of Electrical and Computer Engineering, Rutgers University, North Brunswick, NJ, 08902 USA (e-mail: \{lxiao, ljg, narayan\}@winlab.rutgers.edu).

S. Periyalwar is with SHAPE innovations (e-mail: shalini@ shape.innovations.com).

Digital Object Identifier 10.1109/TCOMM.2008.060330.
The key benefit of the distributed measurement system is that it provides round-the-clock measurements from many low-cost sensors per cell, at known locations. The data so obtained can be used not only for medium-term radio resource management, but also for longer-term engineering, e.g., identifying the need for new cell sites. RF planning for wireless systems utilizes both proactive measurements (e.g., path loss) and reactive measurements (e.g., call drops, handovers). For proactive measurements, in most cases, currently used RF planning tools gather propagation information based on the use of a database in a given region, augmented by drive tests conducted during off-peak times. Such static snapshots of RF planning information are suitable for current systems with fixed antenna patterns and limited use of adaptive algorithms. Data collected by mobiles and relayed to base stations may deliver additional time-of-day-specific RF planning information. However, relying on mobiles alone to provide signal and interference power measurements has limited benefit and adds demands to scarce mobile battery resources. Furthermore, a given mobile can measure downlink conditions only, may not be equipped with GPS receivers to help associate its measurements with location, and reports at uncontrolled times and locations.

Distributed measurements can react to gradual changes in propagation (e.g., new structures, especially in cities) or interference (e.g., due to adaptive beamforming). They are not labor-intensive and are available at all times, to accommodate slow adaptive changes in radio resources. The sensors can be more numerous and measurements may be gathered moreor-less uniformly from known locations, facilitating reliable outage evaluations. In fact, the potential exists to accurately pinpoint chronically poor service areas that arise after initial planning, and to identify the need for new or reengineered sites. Additionally, the sensor network could be extended to support multiple air interfaces within overlapping coverage regions (e.g., wireless LAN, DVB-H deployments).

We envision a network fabric of $N$ sensors per cell $(N \sim$ $100)$ which communicate with each other and, through some sensors, with the cellular system, as shown in Fig. 1. Each sensor has an identifying code and a fixed and known location, and it measures received powers from pilots sent by its closest base and several bases nearby. As we will show, the collection of data from all the sensors can be used to estimate the percentages of each cell having adequate signal-to-noise ratio $(S N R)$, or adequate signal-to-interference ratio $(S I R)$. The percentage of the cell that is not having sufficient $S N R$ (or $S I R$ ) is called the outage probability, $P_{o}$. 


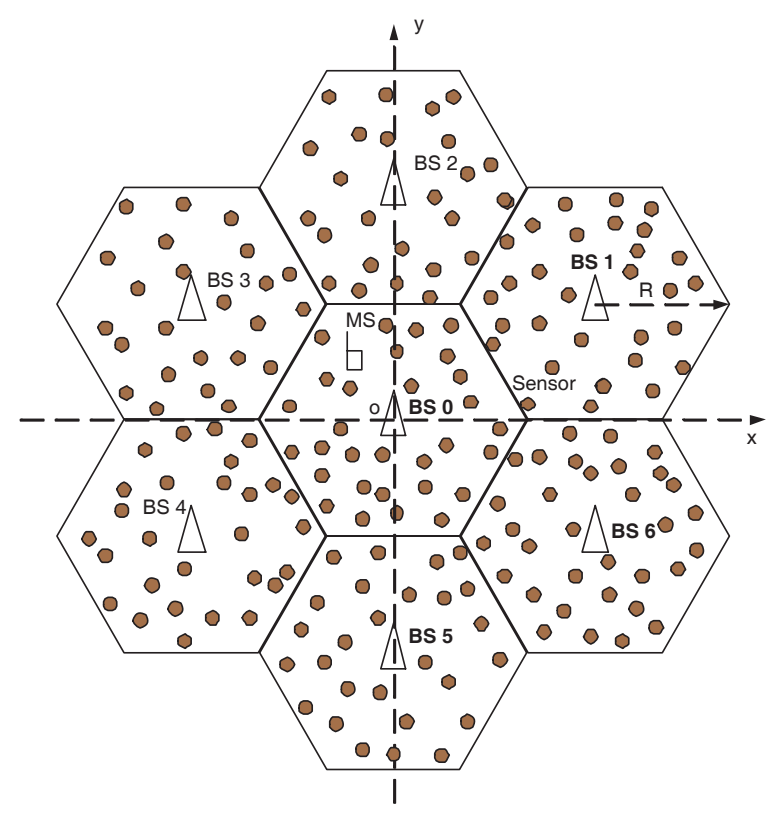

Fig. 1. A 7-cell cluster, with many sensors in each cell. We evaluate "outage" conditions in the center cell, both actual and as estimated using the sensors.

Our calculations are based on a path loss model that incorporates the effects of path length (distance) and spatially correlated shadow fading, as discussed in Section II. We then apply the principle of importance sampling (IS) in the placement of sensors and discuss how it might decrease the number of sensors required for a given accuracy in estimating $P_{o}$ (Section III). Here, we derive an optimal sensor placement strategy for $S N R$-based estimation of $P_{o}$ and also introduce three sub-optimal (but more generic) placement schemes. Next, we describe the use of Monte Carlo simulations to quantify the accuracy in estimating $P_{o}$ as a function of the number of sensors $(N)$, the placement scheme, and various system and path loss model parameters (Section IV). The results, for both $S N R$-based and $S I R$-based estimation, reveal the values of $N$ needed for good accuracy and show the possible reductions in $N$ using $I S$-type sensor placement. We also briefly discuss the use of the distributed measurements to generate outage (or hole) maps. We conclude (Section V) with a general discussion of distributed measurements to assist cell engineering, and we summarize our main findings.

\section{SySTEM MODEL}

We assume each of $N$ sensors in a given cell with radius $R$ measures the received power of a downlink pilot signal. We also assume that the pilot power measurement is over a bandwidth sufficiently wide (5 $\mathrm{MHz}$ or more) that multipath fading is essentially averaged out. Thus, the measurement of received power $P_{R}$, combined with knowledge of the downlink transmit power per user and the antenna gains, permits the network to estimate the downlink path loss, $P L$. For our purposes, it is fair to assume that the antenna gains are independent of sensor position, so that the variation of $P_{R}$ over the sensors precisely tracks the variation of $P L$, i.e., $P_{R}=C-P L$, where $C$ is the same for all sensors. We note that, due to the averaging over multipath fading, this estimate applies to the uplink path loss as well.
Assuming the model of [1], the path loss $(P L)$ from a base station (BS) to a location of the $i$-th sensor in the environment is

$$
P L_{i}[d B]=A+10 \gamma \log \left(d_{i} / d_{o}\right)+s_{i}, \quad d_{i}>d_{o}, i=1, \cdots, N
$$

where $d_{i}$ is the distance from the BS to the $i$-th sensor and $d_{o}$ is a reference distance (typically, $1 \mathrm{~m}$ indoors and 100 m outdoors). The intercept $A$ is given by $20 \log \left(4 \pi d_{o} / \lambda\right)$, where $\lambda$ is the wavelength. The path loss exponent $\gamma$ can range from 3 to 6 , depending on the environment; the $\mathrm{dB}$ shadow fading, $s_{i}$, is a Gaussian process over space with zero mean and standard deviation $\sigma$; and $\sigma$ can range from $4 \mathrm{~dB}$ to $12 \mathrm{~dB}$, depending on the environment. We assume that the autocorrelation of the spatial process $s_{i}$ depends only on the separation distance, i.e.,

$$
E\left[s_{i} s_{j}\right]=\sigma^{2} e^{-d_{i j} / X_{c}}, \quad i, j=1, \cdots, N
$$

where $d_{i j}$ is the distance between the $i$-th and $j$-th sensors; and $X_{c}$, the shadow fading correlation distance, can range from several to many tens of meters [2]. We will assume a frequency of $2 \mathrm{GHz}$ and $\gamma=3.8$ in all our computations ${ }^{1}$; and will consider different combinations of $\sigma, X_{c}, d_{o}$ and cell radius for different cellular environments. We can thus use (1) and (2) to simulate the cell-wide variation of path loss.

For $S N R$-based estimation of outage probability, we compare the path loss, $P L_{i}$, to a threshold value $P L_{0}$. That threshold is the value at which a mobile receiver near the sensor would have just enough fade-averaged signal-to-noise ratio for good reception ${ }^{2}$. A hole in coverage (i.e., an outage) is defined as a location whose path loss from the BS is greater than the threshold, i.e., a hole exists at a location if $P L>P L_{0}$. The fraction of sensors measuring power below the threshold is the sensor network's estimate of the cell's SNR-based outage probability. The SNR-based approach is the same for the downlink and uplink. However the value of $P L_{o}$ may differ for the two directions, due to differences in transmit power, receiver noise level or air interface.

Figure 2 presents an outage map for a circular cell, where the dark spots indicate outages. The outage probability here is $P_{o}=11.8 \%$. Also, $N=4000$ and $P L_{o}=120 \mathrm{~dB}$. The dark outage spots are seen to be concentrated in the outer regions of the cell. Placing sensors close to the center, therefore, can amount to wasting limited resources on what are predictably "non-events". This brings us to the subject of importance sampling [7].

\section{IMPORTANCE SAMPLING (IS) FOR OUTAGE PROBABILITY ESTIMATION}

\section{A. IS Concept and Analysis}

Assume $N$ sensors are placed in the circular cell with radius $R$, and the $i$-th sensor is at a distance $d_{i}$ from the base at an azimuth angle $\varphi_{i}, i=1, \cdots, N$. Assume further

\footnotetext{
${ }^{1}$ While $\gamma$ can have a wide range of values [1], the most common cellular values cited on the literature range from 3.5 [3] to 4.0 [4]; hence, we choose 3.8 for our calculations.

${ }^{2}$ The multipath-averaged SNR is a valid determinant of link performance (e.g., see [5] and [6]), which is why our method is based on fade-averaged measurements.
} 


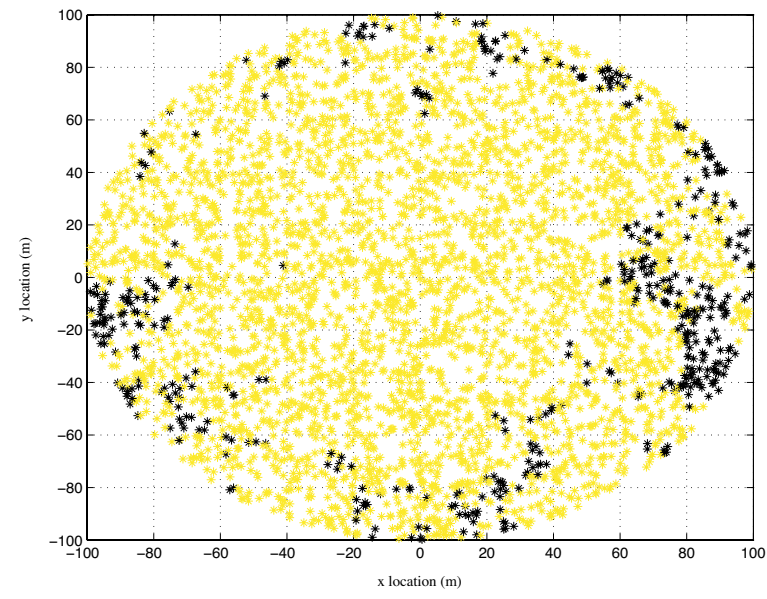

Fig. 2. An "outage" map for a single cell with 4000 uniformly located sensors. In this example, the cell radius is $100 \mathrm{~m}, \sigma=8 \mathrm{~dB}, X_{c}=8 \mathrm{~m}$, and "outage" corresponds to the condition $P L>P L_{o}=120 \mathrm{~dB}$. The dark dots indicate outages, which occur for $11.8 \%$ of all sensors, primarily in sensors located towards the cell boundary.

that each squared distance, $D_{i}=d_{i}^{2}$, is drawn from a set of possible values on $\left[0, R^{2}\right]$ whose underlying probability density function (PDF) is $f(D)$; that each $\varphi_{i}$ has a uniform PDF on $[0,2 \pi]$; and that all the $D_{i}$ and $\varphi_{i}$ are mutually independent. Thus, in populating a given cell with sensors, each sensor can be said to be placed independently and randomly according to the underlying $\operatorname{PDF} f(D)$.

In a traditional Monte Carlo simulation, the sensors would be distributed randomly and uniformly over the full cell; we call this full-cell (FC) placement. Here, $f(D)=1 / R^{2}$ for $0<$ $D<R^{2}$, and zero otherwise. The estimated outage probability is an average over measurements by $N$ sensors, given by

$$
\hat{p}_{F C}=\frac{1}{N} \sum_{i=1}^{N} \phi\left(\xi_{i}\right)
$$

where $\phi(\cdot)$ is an indicator function, i.e., $\phi\left(\xi_{i}\right)=1$ if $P L_{i}>$ $P L_{0}$, otherwise, $\phi\left(\xi_{i}\right)=0$.

Now we consider what happens if we "bias" the placement of the sensors to the area where the outage is more likely to happen. Denote the PDF of $D$ and $d$ in the new placement as $f^{*}(D)$ and $f_{d}^{*}(d)$, respectively. By definition, we have $\int_{0}^{R^{2}} f^{*}(D) d D=1$ and $f^{*}(D) \geq 0$. The estimated outage probability is

$$
\hat{p}_{I S}=\frac{1}{N} \sum_{i=1}^{N} \phi\left(\xi_{i}^{\prime}\right) W\left(\xi_{i}^{\prime}\right)
$$

where $\xi_{i}^{\prime}, i=1, \cdots, N$ is the location of the $i$-th sensor, generated according to $f^{*}(D)$. The weight function $W(\cdot)$ seeks to "undo" the bias due to sampling with the biased placement.

The estimate of outage probability must be unbiased, i.e., $E\left[\hat{p}_{I S}\right]=E\left[\hat{p}_{F C}\right]$. Also, the variance should be smaller, i.e., $\operatorname{Var}\left[\hat{p}_{I S}\right]<\operatorname{Var}\left[\hat{p}_{F C}\right]$, which means that its estimate is sharper than that for full-cell placement with the same amount of sensors, or equivalently, requires fewer sensors for the same estimate sharpness. The average and the variance are taken over different random selections of the sensor positions and the shadow fading realizations. This is the essence of importance sampling [7].

We now consider how to satisfy two conditions: (1) unbiased estimates of $P_{o}$, and (2) minimal estimator variance. To gain insight with minimal complexity, we assume $X_{c}=0$ in the current analysis, i.e., the $\mathrm{dB}$ shadow fadings are are i.i.d Gaussian variables $N\left(0, \sigma^{2}\right)$. In our later computations, we will take shadow fading correlations into account.

Following (1), we write the average outage probability of FC placement over all realizations of the shadow fading as

$$
\begin{aligned}
\hat{P}_{F C} & =\frac{1}{N} \sum_{i=1}^{N} \operatorname{Pr}\left(A+10 \gamma \log \left(d_{i} / d_{o}\right)+s_{i}>P L_{0}\right) \\
& =\frac{1}{N} \sum_{i=1}^{N} Q\left(\frac{P L_{0}-A-10 \gamma \log \left(d_{i} / d_{o}\right)}{\sigma}\right) \\
& =\frac{1}{N} \sum_{i=1}^{N} Q\left(\frac{\beta-\alpha \log \left(D_{i}\right)}{\sigma}\right)
\end{aligned}
$$

where $\alpha=5 \gamma, \beta=P L_{0}-A+10 \gamma \log \left(d_{o}\right)$.

Similarly, we write the average outage probability of IS placement as

$$
\hat{P}_{I S}=\frac{1}{N} \sum_{i=1}^{N} Q\left(\frac{\beta-\alpha \log \left(D_{i}^{*}\right)}{\sigma}\right) W\left(D_{i}^{*}\right)
$$

Condition 1 above amounts to $\left(E\left[\hat{P}_{F C}\right]=E\left[\hat{P}_{I S}\right]=P_{o}\right)$, so we require that

$$
W(D)=\frac{f(D)}{f^{*}(D)}=\left\{\begin{array}{lc}
\frac{1 / R^{2}}{f^{*}(D)}, & 0<D<R^{2} \\
0, & \text { otherwise }
\end{array}\right.
$$

which indicates that $f^{*}(D)>0$ throughout $0<D<R^{2}$.

Condition 2 amounts to minimizing $\operatorname{Var}[\hat{P}]=E\left[\hat{P}^{2}\right]-$ $E^{2}[\hat{P}]$, i.e.,

$$
\begin{aligned}
& \operatorname{Var}_{f^{*}(D)}[\hat{P}]=E_{f^{*}(D)}\left[\hat{P}^{2}\right]-P_{o}^{2} \\
& =\frac{1}{N^{2}} E\left[\sum_{i=1}^{N} \sum_{j=1}^{N} Q\left(\frac{\beta-\alpha \log \left(D_{i}^{*}\right)}{\sigma}\right) W\left(D_{i}^{*}\right)\right. \\
& \left.\quad Q\left(\frac{\beta-\alpha \log \left(D_{j}^{*}\right)}{\sigma}\right) W\left(D_{j}^{*}\right)\right]-P_{o}^{2} \\
& =\frac{1}{N} E_{f^{*}\left(D^{*}\right)}\left[\left(Q\left(\frac{\beta-\alpha \log \left(D^{*}\right)}{\sigma}\right) W\left(D^{*}\right)\right)^{2}\right]-\frac{P_{o}^{2}}{N} \\
& =\frac{1}{N R^{4}} \int_{0}^{R^{2}} \frac{Q^{2}\left(\frac{\beta-\alpha \log (D)}{\sigma}\right)}{f^{*}(D)} d D-\frac{P_{o}^{2}}{N}
\end{aligned}
$$

where the third line follows from the independence of $W\left(D_{i}^{*}\right)$ and $W\left(D_{j}^{*}\right), i \neq j$, and

$$
\begin{aligned}
P_{o} & =E_{f(D)}\left[\hat{P}_{F C}\right]=E_{f *(D)}\left[\hat{P}_{I S}\right]=E\left[Q\left(\frac{\beta-\alpha \log (D)}{\sigma}\right)\right] \\
& \left.=\int_{0}^{R^{2}} Q\left(\frac{\beta-\alpha \log (D)}{\sigma}\right)\right] d D / R^{2}
\end{aligned}
$$

\section{B. Optimal Sensor Placement Scheme}

From the above discussion, we can see that $f^{*}(D)$ for the optimal sensor placement is given by

$$
\begin{array}{ll}
\min & \operatorname{Var}_{f^{*}(D)}[\hat{P}] \\
\text { s.t. } & \int_{0}^{R^{2}} f^{*}(D) d D=1
\end{array}
$$


where $f^{*}(D)>0$ for $0<D<R^{2}$. Its Lagrangian form is

$$
L\left(f^{*}, \lambda\right)=\int_{0}^{R^{2}}\left(Q^{2}\left(\frac{\beta-\alpha \log (D)}{\sigma}\right) / f^{*}(D)+\lambda f^{*}(D)\right) d D
$$

Then the optimization reduces to minimizing $L_{2}\left(f^{*}, \lambda\right)=$ $Q^{2}\left(\frac{\beta-\alpha \log (D)}{\sigma}\right) / f^{*}(D)+\lambda f^{*}(D)$, subject to the constraint in (10). This leads to the following solution for the PDF of $D$ :

$$
f^{*}(D)=\left\{\begin{array}{cl}
Q\left(\frac{\beta-\alpha \log (D)}{\sigma}\right) / \eta, & 0<D<R^{2} \\
0, & \text { otherwise }
\end{array}\right.
$$

where $\eta=\int_{0}^{R^{2}} Q\left(\frac{\beta-\alpha \log (D)}{\sigma}\right) d D$. The corresponding optimal PDF for $d$ is

$$
f_{d}^{*}(x)=\left\{\begin{array}{cl}
2 x Q\left(\frac{\beta-2 \alpha \log (x)}{\sigma}\right) / \eta, & 0<d<R \\
0, & \text { otherwise }
\end{array}\right.
$$

This is a parametric scheme in that it requires knowledge of channel parameters, such as $\sigma$ and $\gamma(=0.2 \alpha)$. Further, this optimal placement essentially requires knowledge of the outage probability $P_{o}$, as can be seen from (9) and the above definition of $\eta$. In traditional IS methods, such an optimal solution is referred to as degenerate, since it requires knowledge of the true value of the quantity being estimated.

\section{Semi-Parametric Placement Schemes}

Motivated by the importance sampling idea above and to overcome the degenerate problem, we propose three sensor placement schemes that are semi-parametric, that is, each exploits the knowledge that power generally falls off with distance but does not attempt to know or exploit the precise nature of that falloff. Each scheme is defined by the PDF it uses for the base-to-sensor distance, $d$; the angular PDF in each case is uniform on $[0,2 \pi]$, as before. The three distance PDFs we consider are the following:

$$
\begin{aligned}
& \text { Scheme 1: } f_{d}(x)=\left\{\begin{array}{cl}
4 x^{3} / R^{4}, & 0<x<R \\
0, & \text { otherwise }
\end{array}\right. \\
& \text { Scheme 2: } f_{d}(x)=\left\{\begin{array}{cl}
6 x^{5} / R^{6}, & 0<x<R \\
0, & \text { otherwise }
\end{array}\right.
\end{aligned}
$$

$$
\text { Scheme } \quad 3: \quad f_{d}(x)=\left\{\begin{array}{cc}
\frac{2 x}{R^{2}-R_{\min }^{2}}, & R_{\min }<x<R \\
0, & \text { otherwise }
\end{array}\right.
$$

Unlike the first two schemes, Scheme 3 is based on the assumption that there are no holes in the inner area of the cell, i.e., at locations with distances smaller than $R_{m i n}$. Here, all the sensors are distributed uniformly in the outer ring of the cell, i.e., at locations with distances between $R_{\text {min }}$ and $R$. Accordingly, we call Scheme 3 partial-cell (PC) placement. From Fig. 3, we see that these PDFs, and especially the one for Scheme 2, are good approximations to the PDF that was shown to be optimal for the case $\gamma=3.8, \sigma=8 \mathrm{~dB}, X_{c}=0$ and $P_{o}=0.05$. How this translates into performance is a topic we address next.

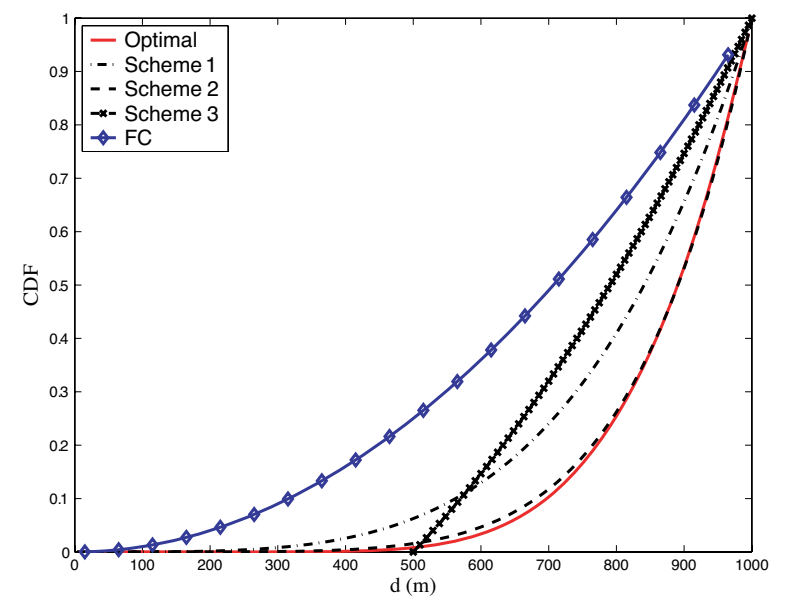

Fig. 3. CDF of $d$ for five sensor placement schemes, including full-cell (FC) placement. The "optimal" scheme is for the case $\gamma=3.8, \sigma=8$ $\mathrm{dB}, X_{c}=0, R=1000 \mathrm{~m}$, and $P_{o}=0.05$. Scheme 3 is partial-cell (PC) placement with $R_{\min }=0.5 R$.

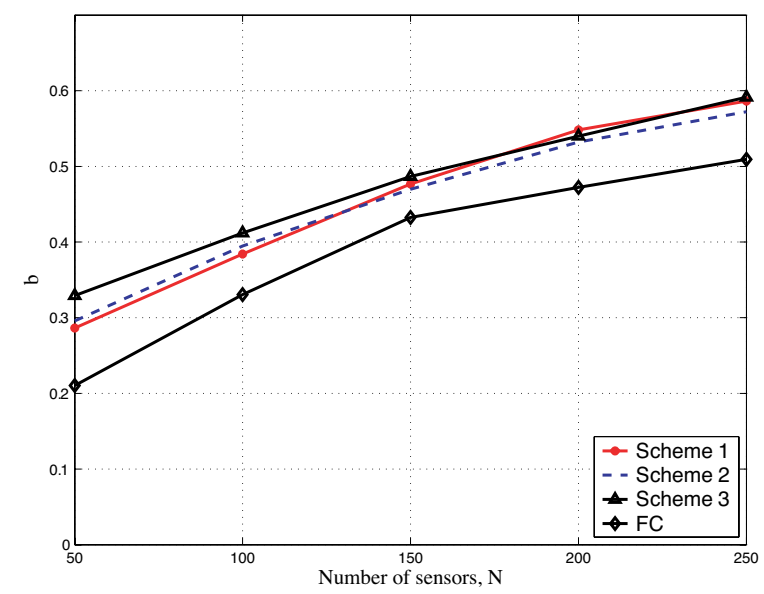

Fig. 4. $b$ vs. $N$, where $b=\operatorname{Pr}\left[\left|\hat{P}_{o}-P_{o}\right|<0.2 P_{o}\right]$, with $R=1000 \mathrm{~m}$, $\gamma=3.8, \sigma=8 \mathrm{~dB}, X_{c}=50 \mathrm{~m}$, and $P_{o}=0.05$.

\section{Simulation RESUlts ON OUTAGE PROBABILITY ESTIMATION}

\section{A. Preliminary Result}

Figure 4 presents the probability that the error in estimating $P_{o}$ falls within $\pm 20 \%$ of the true value, which is 0.05 in this example. Similar results were obtained for $P_{o}=0.10$. It is seen that the three IS-based schemes cited above have similar performance, and all of them are better than full-cell placement. Among the IS-based schemes, Scheme 3 (partialcell placement, (16)) is easy to implement and has very good performance. In the remainder of the paper, we will compare it with full-cell placement for both $S N R$-based and SIR-based outages and for both indoor and outdoor environments.

\section{B. Simulation Approach}

In our simulations, we initially assume a circular cell of radius $R$ (later, we discuss circular vs. hexagonal cell shapes), and we assign values to $R$ and the propagation parameters in (1) and (2). We simulate some number, $N_{s h}$, of statistically similar cells, with each having a different spatial variation of $s$, the $\mathrm{dB}$ shadow fading. We start by simulating $s(\xi)$ for Cell 1 , 
where $\xi$ denotes position within the cell. This random spatial variation is simulated using (2) and Cholesky decomposition, as described in [8]. The next step is to choose a value for $N$, the number of sensors per cell; a placement for the $N$ sensors; and a path loss threshold, $P L_{o}$, the determination of which is described below. Finally, the path loss at each of the sensors is computed, and the outage probability in Cell 1, $p_{o}(1)$, is determined as the fraction of sensors for which $P L>P L_{o}$.

With $s(\xi)$ fixed, the $N$-sensor placement is chosen a total of $M$ times and, if $M$ is sufficiently large, the mean and standard deviation of $p_{o}(1), \mu_{1}$ and $\sigma_{1}$, respectively, can be estimated. (Note that these are the mean and standard deviation taken over the random placements of the $N$ sensors within the cell.) This procedure is repeated for a total of $N_{s h}$ generations of the shadow fading variation $s(\xi)$, corresponding to Cells $1, \ldots, N_{s h}$. The average of $\mu_{j}$ over $j$ is the network's estimate, denoted by $P_{o}^{*}$, of the true outage probability; and the average of $\sigma_{j}$ over $j$, denoted by $\rho$, is the network's estimate of the intra-cell standard deviation due to the random $N$-sensor placement. We call $\rho$ the "sharpness" of the estimate, and seek to make it as small as $0.25 P_{o}$ or less.

The baseline value of $P_{o}$, i.e., what we assume to be the true one, is obtained by first assigning an extremely large value for $N$. We have found, by a combination of analysis and simulation not shown here, that $N=4000$ would yield precise estimates in any cell, with negligible variation from one placement of $N$ sensors to another. For that $N$, we (1) computed outage probability for each of $N_{s h}$ realizations of $s(\xi)$, for each of several values of path loss threshold, $P L_{o}$; (2) averaged over the $N_{s h}$ values for each $P L_{o}$; and (3) took the result to be the "true" outage probability, $P_{o}$, for that path loss threshold. We were thus able to identify the values of $P L_{o}$ producing average outage probabilities of 0.05 and 0.10 . We applied the procedure described in the previous paragraph for each of these $P_{o}$-values, using practical values of $N$ (namely, $50,100,200,300$, and 400). For each of these $N$, we did $M=200$ placements for each of $N_{s h}=10$ realizations of the shadow fading variation, $s(\xi)$.

\section{SNR-Based Outage Probability}

First, we investigate an outdoor system with the usual hexagonal cells, in particular, a center cell and six surrounding cells (Fig. 1). The center cell, for which we will analyze outage probability estimation, is conveniently assumed to be circular, with a radius, $R$, chosen such that the circle has the same area as the actual hexagonal cell. This will simplify analysis with no loss in accuracy; other studies (e.g., [9]) have shown cell shape to be a negligible factor so long as cell area is preserved $^{3}$. The shadow fading parameters $\left(\sigma, X_{c}\right)$ are initially set at $(8 \mathrm{~dB}, 50 \mathrm{~m})$. We set $P L_{o}$ at values that yield "true" outage probabilities, $P_{o}$, of 0.05 and 0.10 . For each of these two values, we have computed the corresponding estimate, $P_{o}^{*}$, for both full-cell sensor placement $\left(R_{\min }=0\right)$ and partial-cell placement $\left(R_{\text {min }}=0.5 R\right.$ and $\left.0.7 R\right)$.

Simulation results for $P_{o}^{*}$ are shown in Table I. The estimates obtained are independent of $N$ except for a slight

\footnotetext{
${ }^{3}$ We note further that the regular hexagon closely approximates a circle, which is why early investigators chose it as the tessellating shape to use in cellular studies [10], [11].
}

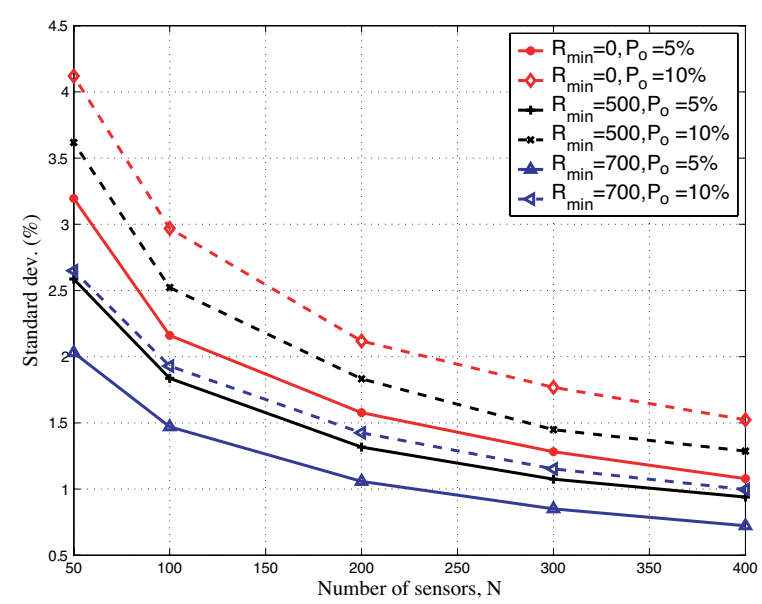

Fig. 5. Standard deviation of estimate for $P_{o}=0.05$ and $0.10, R=1000$ $\mathrm{m}$, and $\left(X_{c}, \sigma\right)=(50 \mathrm{~m}, 8 \mathrm{~dB})$.

fluctuation due to finite simulation, so the numbers shown are averages of those obtained for several values of $N$. We see that the estimation of $P_{o}$ is virtually unbiased for $R_{\min }$ up to at least $0.5 R$, i.e., there are virtually no outages to be counted at smaller distances. At $R_{\text {min }}=0.7 R$, however, the estimation is biased downward because outages can occur at base-terminal distances between $0.5 R$ and $0.7 R$ and are not counted.

For the same case, we obtained simulation results for $\rho$, the average standard deviation of the estimate resulting from the random placement of $N$ sensors. Here, we expect to see a decrease with $N$, as is confirmed by the plots in Fig. 5. The "sharpness" of the estimates improves not only with increasing $N$, but also with increasing $R_{\text {min }}$, because larger $R_{\text {min }}$ leads to a higher density of sensors in the area containing them. A near-best tradeoff between small bias error and maximum "sharpness" occurs for $R_{\min }=0.5 R$, at least for the assumed model. For $\rho$ to be no greater than $0.25 P_{o}$, the figure shows that the required $N$ for $P_{o}=0.05$ is $\sim 200$ for $R_{\min }=0.5 R$ and $\sim 300$ for $R_{\min }=0$ (full placement). For $P_{o}=0.10$, the required $N$-values are $\sim 100$ and 150, respectively. Thus, a simplified form of importance sampling reduces the required number of sensors by about $33 \%$. The general rule is that, with $R_{\min }=0.5 R$, the required $N$ is $\sim 10 / P_{o}$, which is consistent with binomial statistics.

Next, we assume an indoor environment with $R=100$ $\mathrm{m}$, and we consider three different sets of shadow fading parameters $\left(\sigma, X_{c}\right):(8 \mathrm{~dB}, 8 \mathrm{~m}),(8 \mathrm{~dB}, 50 \mathrm{~m})$ and $(10 \mathrm{~dB}$, $50 \mathrm{~m})$. Results are given in Table I and Fig. 6 for $P_{o}=0.10$. These results, and those for $P_{o}=0.05$ as well (not shown), reinforce the findings from the previous example. Moreover, they show that the shadow fading parameters have some influence, but they do not change the general rules for $R_{\min } / R$ and $N$.

\section{SIR-Based Outage Probability}

While the above study of SNR-based outage probability was generic, the study of SIR-based outage probability requires specificity about the radio interface. For this purpose, we assume a CDMA system with a spreading factor of 128 and 


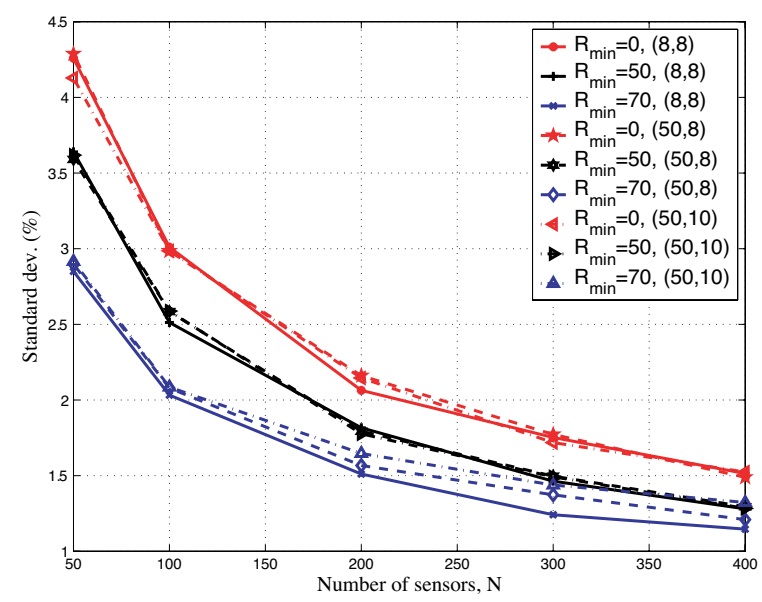

Fig. 6. Standard deviation of estimate for $P_{o}=0.10, R=100 \mathrm{~m}$, and $\left(X_{c}, \sigma\right):(8 \mathrm{~m}, 8 \mathrm{~dB}),(50 \mathrm{~m}, 8 \mathrm{~dB}),(50 \mathrm{~m}, 10 \mathrm{~dB})$.

a required receiver output SIR of $5 \mathrm{~dB}$. For simplicity, we assume that the downlink co-channel interference from the six surrounding cells is dominant. Also, we assume that each sensor is able to identify, from downlink pilots, the power from each base (its own plus the six nearest interfering bases) [12]; that each base is transmitting its full rated power; and that an "outage" occurs for a mobile if its serving base runs out of power before it is able to meet that mobile's SIR requirement. These assumptions, combined with the above path loss model, enable us to compute outage probability for a given number, $K$, of active mobiles per cell (or sector).

We note that for $K>1$, there is one more layer of randomness, besides those for the shadow fading distribution and the sensor placement, namely, the placement of the $K$ mobiles. Thus, for every combination of $s(\xi)$-realization and $N$-sensor placement, the network computes an outage probability for each of $M_{m t}$ random placements of $K$ mobiles over the cell, then averages the $M_{m t}$ values. In our study, we used $M_{m t}=200$.

The above steps are straightforward for full-cell placement $\left(R_{\min }=0\right)$. However, we also considered partial placement, specifically, $R_{\min }=0.5 R$. In this case, the $N$ sensors are uniformly distributed over $3 / 4$ of the cell area, but no sensors are in the inner region $\left(d<0.5 R_{\min }\right)$, where, on average, $1 / 4$ of the $K$ mobiles would be located. This poses a question of how to compute outage probability. To address it, the network can estimate $P_{o}$ as follows: (1) compute an upper bound by assuming all of the $K$ mobiles are in the outer region; (2) compute a lower bound by assuming that $3 / 4$ of the $K$ mobiles are in the outer region and none are in the inner region ${ }^{4}$; and (3) estimate $P_{o}$ as the mean of the two bounds. Our computations show that the upper and lower bound differ from their mean by $3.3 \%, 5.0 \%$ and $6.5 \%$ for $K=4,8$ and 12 , respectively, so the bounds are clearly tight.

Some results are given in Table I and Fig. 7. In Table I, the increase in $P_{o}$ with $K$, due to the dividing of transmit power among more mobiles, is evident but mild. In Fig. 7, we

\footnotetext{
${ }^{4}$ With $K / 4$ mobiles in the inner region, as in an actual $K$-mobile scenario, $P_{o}$ will be higher, due to greater interference; thus, the situation where those $K / 4$ mobiles are absent yields a lower-bound $P_{o}$.
}

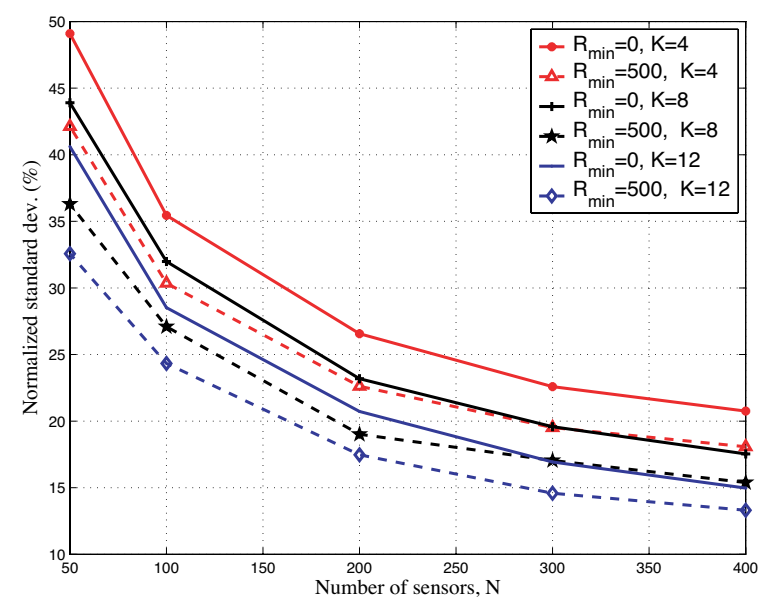

Fig. 7. Normalized standard deviation $\left(\rho / P_{o}\right)$ with $R=1000 \mathrm{~m}, \sigma=8$ $\mathrm{dB}$ and $X_{c}=50 \mathrm{~m}$.

see, as before, that partial placement with $R_{\min }=0.5 R$ and $N \sim 10 / P_{o}$ yields the desired level of "sharpness".

Extensions of this case might take into account issues such as base station selection (e.g., SIR-based as opposed to distance-based, as assumed here), other kinds of interference (outer rings, intra-cell, etc), location information and uplink performance. Regarding the latter, we note that each sensor is assumed to be able to estimate its path loss (which is essentially the same in both directions) to the seven nearest bases. This information, plus some additional computing, would allow the sensor network to estimate uplink outage probabilities, as well. The availability of location information can be used to map the variations in outage regions, as we briefly discuss next.

\section{E. Outage Maps}

Here, we consider how to draw an "outage map" using data collected by the sensors. Specifically, given the quality of the links to $N$ locations, we would like a map that shows or predicts most of the outage locations within a cell. One possibility is to center a circular area ("neighborhood") with radius $R_{c}$ about each sensor that detects an outage. The neighborhoods so identified can be considered to be outage regions requiring some sort of engineering attention.

Figure 8 illustrates one such example, where $N=200$ sensors are used and $R_{c}=100 \mathrm{~m}$ in a cell of $1-\mathrm{km}$ radius. All diamonds represent sensor locations; all stars represent outage locations (out of the 4000 locations from which the sensor locations are chosen); and each circle denotes an outage "neighborhood", at the center of which is a sensor that detects and declares an outage. All stars outside of the circles denote outage locations that are not detected.

Figure 9 exemplifies the average percentage of outage locations detected as a function of $R_{c} / R$, using partial-cell placement with $R_{\min }=0.5 R$. The averaging in these results is over both sensor placements and shadow fading realizations; and the outages are SNR-based. The parameters varied are the shadow fading correlation distance and the number of sensors. Other conditions assumed are given in the figure caption.

We see that the percentage of holes detected increases with $N, X_{c}$ and $R_{c} / R$, though the rate of improvement falls with 
TABLE I

Estimated Downlink OUtage Probability.

\begin{tabular}{|c||c|c|c|}
\hline CASE & $R_{\min }=0$ & $R_{\min }=0.5 R$ & $R_{\min }=0.7 R$ \\
\hline \hline \multicolumn{3}{|c|}{ SNR-Based; Outdoor Cell $(R=1000 \mathrm{~m}) ;\left(\sigma, X_{c}\right)=(8 \mathrm{~dB}, 50 \mathrm{~m})$} \\
\hline "True" $P_{o}=0.05$ & 0.050 & 0.050 & 0.044 \\
\hline "True" $P_{o}=0.10$ & 0.100 & 0.098 & 0.086 \\
\hline \hline \multicolumn{4}{|c|}{ SNR-Based; Indoor Cell $(R=100 \mathrm{~m}) ;$ "True" $P_{o}=0.10$} \\
\hline$\left(\sigma, X_{c}\right)=(8 \mathrm{~dB}, 8 \mathrm{~m})$ & 0.100 & 0.099 & 0.089 \\
\hline$\left(\sigma, X_{c}\right)=(8 \mathrm{~dB}, 50 \mathrm{~m})$ & 0.099 & 0.100 & 0.092 \\
\hline$\left(\sigma, X_{c}\right)=(10 \mathrm{~dB}, 50 \mathrm{~m})$ & 0.100 & 0.099 & 0.089 \\
\hline \hline SIR-Based; Outdoor Cell $(R=1000 \mathrm{~m}) ;$ CDMA System; $\left(\sigma, X_{c}\right)=(8 \mathrm{~dB}, 50 \mathrm{~m})$ \\
\hline$K=4\left(P_{o}=0.076\right)$ & 0.075 & 0.074 & - \\
\hline$K=8\left(P_{o}=0.088\right)$ & 0.088 & 0.087 & - \\
\hline$K=12\left(P_{o}=0.103\right)$ & 0.103 & 0.100 & - \\
\hline
\end{tabular}

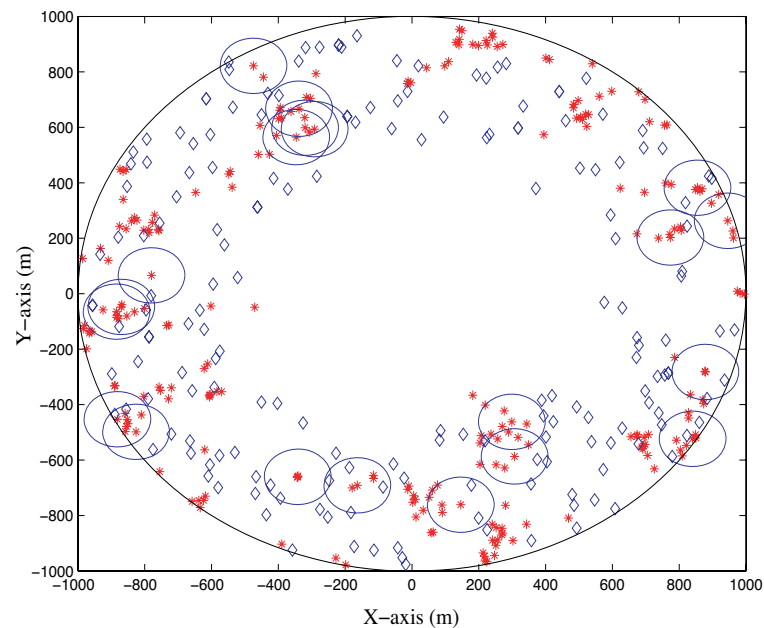

Fig. 8. An "outage map" for a single cell using data collected by $N=200$ sensors. In this example, the cell radius $R=1000 \mathrm{~m}, R_{c}=100 \mathrm{~m}, \sigma=8$ $\mathrm{dB}, X_{c}=50 \mathrm{~m}$. All diamonds represent sensor locations; all stars represent outage locations; and each circle denotes an outage "neighborhood", at the center of which is a sensor that detects and declares an outage. All stars outside of the circles denote outage locations that are not detected.

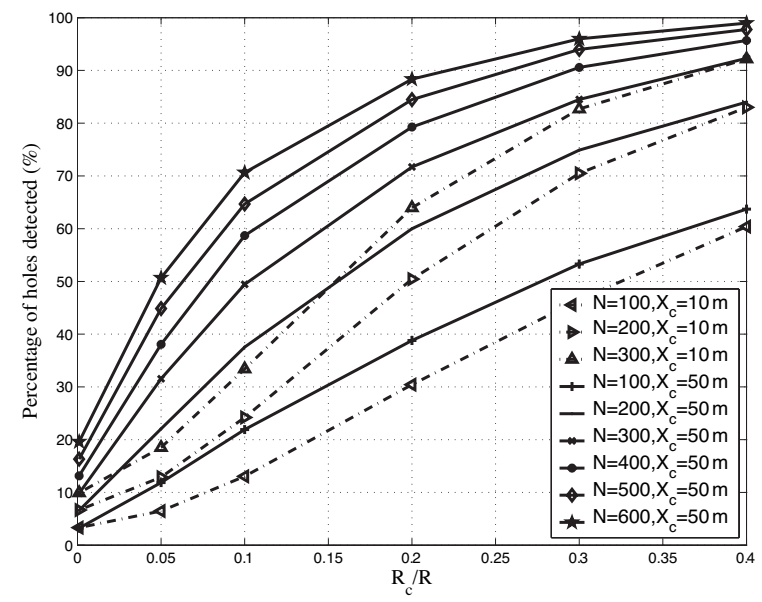

Fig. 9. Percentage of holes detected by $N$ sensors in partial-cell placement, with $R_{\text {min }}=0.5 R, P_{o}=0.05, \sigma=8 \mathrm{~dB}, \gamma=3.8, R=1000 \mathrm{~m}$, and various combinations of $N$ and $X_{c} . R_{c}$ ranges from $1 \mathrm{~m}$ to $400 \mathrm{~m}$.

$N$. Also note that the interesting region of values of $R_{c} / R$ is the low end, typically 0.1 and below, where a "neighborhood" is no bigger than $\sim 1 \%$ of the cell area. In this range, the difference between correlation distances of $10 \mathrm{~m}$ and $50 \mathrm{~m}$ can be substantial. Also, 200 sensors is not nearly enough to capture, on average, the majority of the outages. For that purpose, at least 300 sensors are needed, and as many as 600 are needed to detect $70 \%$ of all outages. To detect that percentage using $\sim 200$ sensors, the sensor data would have to be augmented by other information, such as topographical maps, building location data, and so on.

\section{DisCUSSION AND CONCLUSION}

We applied the principle of importance sampling to improve the efficiency of the distributed measurements in cellular systems. Specifically, we investigated ways to minimize the number $(N)$ of sensors needed to estimating the signal coverage of the cells. We derived, for a particular set of parameters, an optimal sensor placement scheme for estimating cell outage probability; and we used it to postulate schemes that require no specific parameter information. Among them, we emphasize a version of partial-cell placement, wherein the power-measuring sensors are distributed in a random uniform way over base-mobile distances from $50 \%$ to $100 \%$ of the cell radius. Its performance was compared with that of fullcell placement. It was shown that a cell outage probability of $P_{o}$ can be accurately estimated using $\sim 10 / P_{o}$ sensors with partial-cell placement; and that this represents a reduction, relative to full-cell placement, of $\sim 33 \%$. This result applies to both $S N R$-based and $S I R$-based outage estimation for both indoor and outdoor environments. We also presented a way to draw outage maps based on measurement data from the sensors, and showed that many more sensors are required to capture most of a cell's outage locations.

While we have focused in this paper on sensor-based information, a given operator may want to consider a wide range of approaches, including: (1) The traditional combining of site data with drive testing; (2) deploying a dedicated network of sensors; (3) renting service from an existing multipurpose sensor network; (4) using a set of subscriber mobiles, equipped with GPS, to periodically measure and report power measurements; and so on. For those approaches based on sensor or mobile measurements, the rate of measurement-andreport (e.g., hourly, daily, etc) can be tailored to maintain acceptable levels of battery drain. Choosing among candidate approaches would require a cost/performance tradeoff analysis that is beyond the scope of this study; our main purpose here has been to demonstrate that accurate outage estimation based on distributed power measurements is possible and moreover, that the required number of such measurements can be substantially reduced via importance sampling. 


\section{REFERENCES}

[1] V. Erceg and et al, "An empirically based path loss model for wireless channels in suburban environments," IEEE J. Select. Areas Commun., vol. 17, pp. 1205-1211, July 1999.

[2] M. Gudmundson, "Correlation model for shadow fading in mobile radio systems," Electron. Lett., vol. 27, pp. 2145-2146, Nov 1991.

[3] M. Hata, "Empirical formula for propagation loss in land mobile radio services," IEEE Trans. Veh. Technol., pp. 317-325, Aug. 1980.

[4] A. F. Molisch, Wireless Communications. New York: John Wiley and Sons, 2005, ch. 3.2.1.

[5] ibid, ch. 4.2.3.

[6] A. Goldsmith, Wireless Communications. Cambridge, UK: Cambridge University Press, 2005.

[7] P. Hahn and M. Jeruchim, "Developments in the theory and application of importance sampling," IEEE Trans. Commun., vol. 35, pp. 706-714, July 1987.

[8] T. Kailath, A. H. Sayed, and B. Hassibi, Linear Estimation. Englewood Cliffs, NJ: Prentice-Hall, 2000.

[9] S. Kishore, L. J. Greenstein, H. V. Poor, and S. C. Schwartz, "Uplink capacity in a cdma macrocell with a hotspot microcell: exact and approximate analyses," IEEE Trans. Wireless Commun., vol. 2, pp. 364 374, Mar. 2003

[10] W. C. Jakes, Microwave Mobile Communications. Piscataway, NJ: IEEE Press, 1994, ch. 7.2.1.

[11] V. H. MacDonald, "The cellular concept," Bell Sys. Tech. J., vol. 58, pp. 15-41, Jan. 1979.

[12] H. Holma and A. Toskala, WCDMA for UMTS-Radio Access for Third Generation Mobile Communications. New York: John Wiley \& Sons, 2000, ch. 6 .

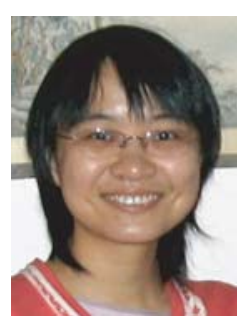

Liang Xiao received the B.S. in communication engineering in 2000 from Nanjing University of Posts \& Telecommunications, Nanjing, China, and the M.S. in electrical engineering in 2003 from Tsinghua University, Beijing, China. From 2003 and 2004, she was with North Carolina State University, NC. She is currently working toward the Ph.D. degree in WINLAB, Department of Electrical and Computer Engineering, Rutgers University, NJ.

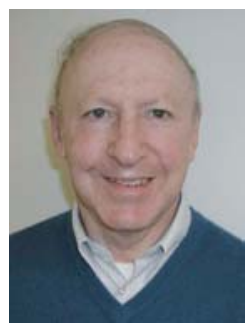

Larry J. Greenstein received the B.S., M.S., and $\mathrm{PhD}$ degrees in electrical engineering from Illinois Institute of Technology, Chicago, IL, in 1958, 1961, and 1967, respectively. From 1958 to 1970 , he was with IIT Research Institute, Chicago, IL, working on radio frequency interference and anti-clutter airborne radar. He joined Bell Laboratories, in Holmdel, NJ, in 1970. Over a 32-year AT\&T career, he conducted research in digital satellites, point-to-point digital radio, optical transmission techniques, and wireless communications. For 21 years during that period (1979-2000), he led a research department renowned for its contributions in these fields. He is now a Research Scientist at Rutgers-WINLAB, North Brunswick, NJ, working in the areas of ultra-wideband (UWB), sensor networks, MIMO-based systems, Broadband Power Line systems and radio channel modeling.

Dr. Greenstein is an AT\&T Fellow, recipient of the IEEE Communications Society's Edwin Howard Armstrong Award, and winner of three best paper awards. He is currently the Director of Journals for the IEEE Communications Society.

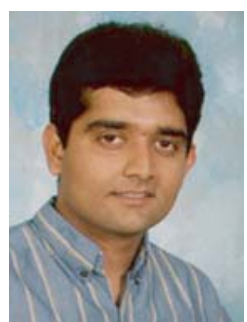

Narayan B. Mandayam received the B.Tech (Hons.) degree in 1989 from the Indian Institute of Technology, Kharagpur, and the M.S. and Ph.D. degrees in 1991 and 1994 from Rice University, Houston, TX, all in electrical engineering. From 1994 to 1996, he was a Research Associate at the Wireless Information Network Laboratory (WINLAB), Dept. of Electrical \& Computer Engineering, Rutgers University. In September 1996, he joined the faculty of the ECE department at Rutgers where he became Associate Professor in 2001 and Professor in 2003. Currently, he also serves as Associate Director at WINLAB. He was a visiting faculty fellow in the Department of Electrical Engineering, Princeton University in Fall 2002 and a visiting faculty at the Indian Institute of Science in Spring 2003.

His research interests are in various aspects of wireless data transmission including system modeling and performance, signal processing and radio resource management with emphasis on techniques for cognitive radio technologies.

Dr. Mandayam is a recipient of the Institute Silver Medal from the Indian Institute of Technology, Kharagpur in 1989 and the National Science Foundation CAREER Award in 1998. He was selected by the National Academy of Engineering in 1999 for the Annual Symposium on Frontiers of Engineering. $\mathrm{He}$ is a coauthor with C. Comaniciu and H. V. Poor of the book Wireless Networks: Multiuser Detection in Cross-Layer Design (Springer, NY). He has served as an Editor for the journals IEEE Communication Letters (19992002), IEEE Transactions on Wireless Communications (2002-2004) and as a guest editor of the 2007 IEEE JSAC Special Issue on Adaptive, Spectrum Agile and Cognitive Radio Networks. He is currently serving as a guest editor of the IEEE JSAC Special Issue on Game Theory in Communication Systems.

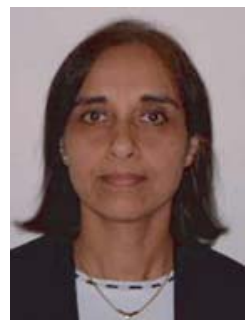

Shalini Periyalwar received her B.Eng. degree in electrical engineering from UVCE, Bangalore University, India, and her MASc and PhD degrees in electrical engineering from Technical University of Nova Scotia, Halifax, Canada. From 1991-1994, she held an industry funded research position as Assistant Professor in the Department of Electrical Engineering at the Technical University of Nova Scotia (now Dalhousie University) with research focus on physical layer design for the wireless air interface. From 1994-2006, Dr. Periyalwar was employed with Nortel, where she held technical management positions and system architect positions for advanced wireless system design. Since 1998, she has held an Adjunct Research Professor position in the Department of Systems and Computer Engineering at Carleton University. Dr. Periyalwar is currently a principal and consultant at SHAPE innovations inc., specializing in advanced wireless technologies and applications. Dr. Periyalwar has over 20 patents and over 30 refereed technical papers in the area of wireless communications. Her current research interests are in the areas of multihop cognitive wireless networks, optimizing delivery of broadcast content in heterogeneous networks and wireless applications for vertical markets. Dr. Periyalwar is a Senior Member of IEEE. 